\title{
Aproximación a los Cuidados Paliativos en las enfermedades avanzadas no malignas
}

\author{
R. NAVARRO SANZ, C. LÓPEZ ALMAZÁN \\ Área Médica Integral. Hospital Pare Jofré. Agencia Valeciana de Salud. Conselleria de \\ Sanitat. Valencia
}

\author{
APPROXIMATION TO PALLIATIVE CARE IN THE ADVANCED NON- \\ MALIGNANT DISEASES
}

\section{RESUMEN}

En la actualidad la Medicina Paliativa (MP) está cambiando desde una visión específica dirigida a los pacientes con cáncer avanzado hacia otra más genérica que abarca también a los pacientes con enfermedades avanzadas no malignas. También está cada vez más arraigado que los cuidados al final de la vida constituyen un derecho fundamental de la sociedad. Pero en realidad estos pacientes no oncológicos excepcionalmente entran en programas de Cuidados Paliativos (CP). Se sabe que es por la dificultad en diagnosticar la situación clínica de enfermedad terminal (SCET). En el presente artículo comentaremos la justificación y limitación de los CP en los pacientes no oncológicos así como la situación paradójica que tiene lugar a pesar del incremento paulatino de los programas de cuidados paliativos. Pero sobre todo proponemos una manera práctica de poder determinar cuando un paciente con enfermedad avanzada de órgano no maligna puede ser subsidiario de unos adecuados $\mathrm{CP}$. Para ello tenemos que conocer el diagnóstico y los factores pronósticos relacionados con la SCET de las patologías crónicas avanzadas de órgano más frecuentes (enfermedad pulmonar crónica avanzada, insuficiencia cardiaca crónica avanzada, cirrosis hepática avanzada, insuficiencia renal crónica avanzada y demencias muy evolucionadas), establecer una adecuada toma de decisiones teniendo en cuenta las preferencias o deseos del paciente y familia, documentar y registrar en la historia clínica todos estos parámetros y ofrecer al paciente el tratamiento más adecuado con el fin de conseguir una muerte digna y considerando unos estándares clínicos, culturales y éticos.

Se destaca la necesidad de llevar a cabo estudios prospectivos que ayuden a establecer unos criterios de inclusión en programas de $\mathrm{CP}$ a pacientes con enfermedades avanzadas de órgano no malignas. Palabras clave: Cuidados Paliativos, Medicina Paliativa, Cuidados al final de la vida, Cuidados Paliativos no oncológicos

PALABRAS CLAVE: Cuidados Paliativos. Medicina Paliativa. Cuidados al final de la vida. Cuidados Paliativos no oncológicos.

\section{ABSTRACT}

Nowadays Palliative Medicine (PM) is changing from a specific point of view towards patients with advanced cancer, to another more generic that also keep in mind patients with advanced non malignant disease. Likewise it is more and more deeply rooted customs that the end-oflife care has become a fundamental right of our Society. But as a matter of fact, these patients with non-cancer diseases unusually go into a Palliative Care $(P C)$ programme. It is known the difficulty to diagnosis the end of life clinical condition (EOLCC) in them. In this article we comment the justification and restriction of $P C$ in patients with non-malignant cancer diseases, as well as the paradoxical situation to come out, in spite of the increasing programes of PC gradually. But above all we propose in a practical way resolve when a patient with non malignant organ advanced disease (NMOAD) could be subsidiary of PC. For that purpose we have to know the diagnosis and the prognostic factors in connection with the EOLCC of the NMOAD more common (advanced chronical pulmonary disease, advanced chronical heart failure, advanced cirrhosis hepatic, advanced chronical renal failure and very evolved dementia), to set up an appropriate make decisions keeping in mind the preferences and wishes of the patient and family, to document and record in the clinical history all those parameters and offerer to the patient the treatment more suitable with the intention to get a worthy death bearing in mind clinical, cultural and ethical standards.

It is pointed out the necessity to carry out prospective studies to help setting up some inclusion criterions in PC programmes for patients with NMOAD.

KEY WORDS: Palliative Care. Palliative Medicine. End-of-life Care. Non oncologic Palliative Care.

Navarro Sanz R, López Almanzán C. Aproximación a los Cuidados Paliativos en las enfermedades avanzadas no malignas. An Med Interna (Madrid) 2008; 25: 187-191.

\section{INTRODUCCIÓN}

Los CP han demostrado su eficacia a la hora de aumentar la calidad de vida de los pacientes oncológicos en SCET y son plenamente aceptados y valorados positivamente tanto por ellos como por sus familias (1). Sin embargo está demostrado que los pacientes con enfermedades crónicas no oncológicas en fase terminal presentan un grado de distress sintomático

Trabajo aceptado: 15 de noviembre de 2007

Correspondencia: Ramón Navarro Sanz. Área Médica Integral. Hospital Pare Jofré. Agencia Valenciana de Salud. Conselleria de Sanitat. C/ San Lázaro, s/n. 46017 Valencia. e-mail: navarro_ram@gva.es 
semejante al de los pacientes oncológicos y se pueden beneficiar de los programas de CP (2-5). La MP está cambiando desde una visión específica para los pacientes de cáncer avanzado a otra más genérica que abarca también a los pacientes con enfermedades avanzadas no malignas como la esclerosis múltiple, enfermedad de motoneurona, SIDA, enfermedades avanzadas de órgano, demencias, etc. (6). Además tenemos que tener siempre presente que el morir con dignidad es reconocido por los profesionales de la salud como uno de los derechos humanos fundamentales (7). Hoy en día es mejor hablar de situaciones clínicas al final de la vida, donde la enfermedad terminal se encuentra entre enfermedad incurable avanzada y la situación de agonía $(8,9)$.

Los objetivos del presente artículo son: comentar la justificación y limitación de los CP en pacientes con enfermedades crónicas avanzadas no malignas, así como la situación paradójica a la hora de aplicar los CP en estos pacientes y sobre todo hacer un intento práctico de resolver dicha situación con el fin de poder determinar cuando un paciente con enfermedad avanzada de órgano no maligna es subsidiario de aplicar unos $\mathrm{CP}$ adecuados.

\section{RAZONES QUE JUSTIFICAN LOS CUIDADOS PALIATIVOS NO ONCOLÓGICOS}

Existen razones que justifican la necesidad de los CP en los pacientes con enfermedades terminales no malignas a pesar de las dificultades que entraña establecer un claro pronóstico de vida. Destacamos: a) el envejecimiento de la población, sobre todo de los ancianos $>85$ años, que conllevará en los próximos años un aumento de la mortalidad por enfermedades crónicas progresivas (10); b) la mortalidad en las personas mayores de 65 años predomina en el grupo de las enfermedades crónicas avanzadas no malignas y solo el $20 \%$ de las causas de muerte es por enfermedad oncológica (11); c) en muchos de los Pacientes Ancianos Frágiles que tras larga enfermedad no responden a los tratamientos específicos, aparece un fallo multiorgánico, progresan los síntomas y además existe un deterioro paulatino funcional global que les puede conducir a una SCET y necesidad de CP (12); d) desde finales del pasado siglo se viene apreciando una serie de fenómenos sociales: aumento del interés en las personas mayores por "morir con dignidad", la legalización del “Testamento Vital", la extensión de la filosofía de los CP a todos los grupos de edad (13); e) el Health Care Financing Administration (HCFA), tiene aprobado un nuevo código de diagnóstico para CP que está incluido en la International Classification of Diseases, 9th Revisión desde 1/10/1996 y como consecuencia ha aparecido un nuevo Grupo Relacionados con el Diagnóstico (GRD) que permite conocer el gasto durante la etapa final de la vida de los pacientes que mueren en programas de CP (14); f) el aumento del consumo recursos de los pacientes en el último año de vida; así en USA el gasto por la atención sanitaria al final de la vida supone el $27 \%$ del presupuesto del Medicare. Dicho gasto en las personas mayores en el último año de sus vidas es un $274 \%$ mayor que el de las personas de su mismo grupo etario que no fallecen en ese año. En Inglaterra, el 22\% de las camas/día hospitalarias están ocupadas por pacientes en el último año de sus vidas (15).

\section{LIMITACIONES DE LOS CUIDADOS PALIATIVOS}

En la mayoría de los países avanzados los CP no cubren las necesidades de la mayoría de los pacientes terminales. Ello se debe a una serie de hechos que vienen referenciados en la literatura médica y que destacamos (14):

-Disponibilidad limitada de los recursos de CP (solo el $17 \%$ de los pacientes terminales están en programas de $\mathrm{CP}$ ).

- Muchos médicos tienen dificultades para hablar a los pacientes sobre la terminalidad de la enfermedad porque piensan que les están quitando esperanza a los propios pacientes.

- Necesidad de estar relativamente seguros sobre la corta supervivencia. De allí la ayuda que pueden proporcionar los indicadores pronósticos sobre todo para los pacientes con enfermedades avanzadas no malignas.

- Necesidad de mayor difusión y enseñanza de los principios básicos de la medicina paliativa a los profesionales de la salud. El estudio SUPPORT ya demostró que: 50\% de los médicos no conocen o no hacen mención sobre "advances directives" (directrices adelantadas), ordenes de resucitación cardiorespiratoria (RCP) hasta 24 horas antes de la muerte de los pacientes y el $40 \%$ de los pacientes tienen dolor potencialmente tratable durante días antes de la muerte (16).

\section{SITUACIÓN PARADÓJICA}

Ya hemos comentado como los CP han demostrado su eficacia en los pacientes oncológicos en situación de enfermedad terminal y son plenamente aceptados en su aplicación a pacientes con enfermedades crónicas avanzadas no malignas. Pero sin embargo, se da el hecho de que estos pacientes no oncológicos, que por unas u otras causas se encuentran en situación de enfermedad avanzada, son aceptados excepcionalmente en las unidades de CP a pesar de que existe un crecimiento en el número de las mismas (en 1999 existían 6.560 Hospices o servicios de CP en 84 países; lo que suponía una media de 78 unidades por país. En el Reino Unido un $70 \%$ pacientes con cáncer podían acceder a un equipo de CP. En 1995, solo 3,3\% ingresos en unidades de CP eran pacientes con diagnóstico de enfermedad no oncológica. Además existe una limitación al acceso a CP más evidente en pacientes en SCET de áreas sociales más deprimidas y en pacientes mayores) $(1,17,18)$.

Nos encontramos ante una situación paradójica que viene dada ante el hecho de un incremento del número de unidades y programas de CP más una mayor morbilidad y mortalidad por enfermedades crónicas progresivas y una limitación para poder aplicar los CP a estos pacientes. La explicación de esta situación paradójica podemos encontrarla en (5):

-Que los CP históricamente se han desarrollado en el ámbito de la oncología; b) la dificultad en identificar la SCET en los pacientes con enfermedades crónicas avanzadas no malignas dada la heterogeneidad de estos enfermos y a la imprecisión a la hora de establecer un pronóstico de vida de alrededor de 6 meses; lo que conlleva a un retraso en el inicio del tratamiento paliativo $(3,5,19)$; c) el hecho de que las etapas finales de las enfermedades crónicas avanzadas son asumidas por las diferentes especialidades médicas. Pero como señala el estudio SUPPORT no siempre existe 
formación en CP ni costumbre de hablar con el paciente y familia acerca de los directrices anticipadas, toma de decisiones, etc. (16).

Tenemos que hacer el esfuerzo necesario para intentar modificar esa situación paradójica ya que va contra los principios básicos de la bioética de la práctica médica (justicia, beneficiencia, no maleficiencia, solidaridad y humanidad) (20).

ENFOQUE PRÁCTICO PARA PODER PROPORCIONAR UNOS CP ADECUADOS A PACIENTES CON ENFERMEDADES AVANZADAS NO MALIGNAS

Es conocida la dificultad que existe para determinar cuando un paciente con enfermedad avanzada no maligna entra en fase terminal; y la razón principal radica en establecer un pronóstico de vida de alrededor de seis meses y en el grado de heterogeneidad que presentan $(3,5,19)$. Estos pacientes con enfermedades crónicas tienen un comienzo gradual de los síntomas, largos períodos de evolución en los que presentan exacerbaciones y una fase de declive no claramente identificable (demencias con períodos largos de enfermedad terminal, insuficiencias cardíacas o respiratorias crónicas avanzadas con cambios clínicos bruscos y continuos, etc. ) (21).

En la práctica clínica es importante, para poder vencer esa dificultad diagnostica y proporcionar unos cuidados adecuados al final de la vida, tener en cuenta los siguientes tres apartados (22):

- Determinar y comunicar el diagnóstico y el pronóstico. Una vez el diagnóstico es conocido, el llegar a establecer una estimación del pronóstico es importante para los pacientes y médicos con vistas a programar una adecuada toma de decisiones y así proporcionar unos cuidados paliativos adecuados (23). Predecir la supervivencia en medicina no es una ciencia precisa, lo que hace difícil poder determinar cuando un paciente con enfermedad avanzada no maligna entra en fase terminal. Por ello es fundamental realizar una Valoración Integral individualizada con herramientas para ajustar el pronóstico (Escalas de valoración de las Actividades Básicas e Instrumentales de la vida diaria, exámenes cognitivos como los tests de Pfeiffer y Folstein, escalas de depresión, etc.) (24). Existen otras variables que nos ayudan junto con la valoración a establecer el pronóstico: a) la edad como indicador de comorbilidad y "fragilidad" (El síndrome de Declive es, en muchos casos, una situación que proviniendo desde la fragilidad acabará conllevando situaciones irreversibles); b) el tiempo de evolución del proceso causante de discapacidad; c) el estado nutricional; d) el deterioro cognitivo; e) la depresión; y f) la falta de un adecuado soporte sociofamiliar (25-31)

Destacaremos a continuación los factores pronóstico relacionados con la SCET de las enfermedades crónicas avanzadas de órgano más frecuentes:

- Enfermedad pulmonar crónica avanzada (32,33): a) Enfermedad pulmonar crónica severa documentada por disnea de reposo con respuesta escasa o nula a broncodilatadores; b) Progresión de la enfermedad evidenciada por: Incremento de las hospitalizaciones o visitas domiciliarias por infecciones respiratorias y/o insuficiencias respiratorias; c) Hipoxemia, $\mathrm{pO}_{2} \leq 55 \mathrm{mmHg}$ en reposo y respirando aire ambiente o $\mathrm{StO}_{2}$ $\leq 88 \%$ con $\mathrm{O}_{2}$ suplementario, o hipercapnia, $\mathrm{pCO}_{2} \geq 50$
$\mathrm{mmHg}$; d) Insuficiencia cardíaca derecha secundaria a enfermedad pulmonar; e) Pérdida de peso no intencionada de $>$ $10 \%$ durante los últimos seis meses; y f) Taquicardia de $>100$ ppm en reposo.

Estos criterios hacen referencia a pacientes con varias formas de enfermedad pulmonar avanzada que siguen a un final común de enfermedad pulmonar terminal. Los criterios a, b y c, deben estar presentes.

- Insuficiencia cardiaca crónica avanzada (34,35): a) Disnea grado IV de la New York Heart Association (NYHA); b) Fracción de eyección $\leq$ del 20\%; c) Persistencia de los síntomas de insuficiencia cardíaca congestiva a pesar del tratamiento adecuado con diuréticos, vasodilatadores e IECAs; y d) Insuficiencia cardíaca refractaria y arrítmias supraventriculares o ventriculares resistentes al tratamiento antiarrítmico.

La situación clínica de terminalidad viene dada cuando la insuficiencia cardíaca avanzada es refractaria al tratamiento médico y no es subsidiaria de trasplante cardíaco.

- Enfermedad hepática avanzada: Cirrosis hepática (36). a) Insuficiencia hepática grado $\mathrm{C}$ de la clasificación de ChildPugh: Encefalopatía grado III-IV, ascitis masiva, bilirrubina $>$ $3 \mathrm{mg} / \mathrm{dl}$, albúmina $<2,8 \mathrm{~g} / \mathrm{dl}$, tiempo de protrombina $<30 \%$; y en la que se ha descartado el trasplante hepático; y b) El síndrome hepatorrenal debido a que carece de tratamiento médico eficaz ya suele ser un indicador de situación clínica terminal.

- Insuficiencia renal crónica avanzada (37). La situación terminal en pacientes con enfermedad renal avanzada y que no van a ser dializados viene dada ante: a) manifestaciones clínicas de uremia (confusión, naúseas y vómitos refractarios, prurito generalizado, etc.; b) diurésis < 400 cc/día; c) hiperkaliemia $>7$ y que no responde al tratamiento; 4) pericarditis urémica; d) síndrome hepatorrenal; y e) sobrecarga de fluídos intratable.

- Demencias muy evolucionadas (38). a) Functional Assessment Staging (FAST) > 7c; b) deterioro cognitivo severo; c) dependencia absoluta; d) presencia de complicaciones (comorbilidad, infecciones de repetición -urinarias, respiratorias-, sepsis, fiebre a pesar de la antibioterapia, etc....); e) disfagia; y f) desnutrición.

- Establecer preferencias personales para cada caso. Existe escasa documentación sobre la aceptación de las metas y preferencias de los pacientes en los cuidados al final de la vida. En el estudio SUPPORT solo el $60 \%$ de los médicos que habían recibido dicha información relatan que la tenían y solo un tercio vuelven a solicitar información sobre las preferencias de los pacientes. Existe escasa cultura médica sobre el aceptar los deseos de los pacientes. Del citado estudio también se conoce que casi la mitad de las órdenes de no RCP fueron documentadas dos días antes de la muerte y escasamente la mitad de los pacientes que preferían no RCP tenían las órdenes escritas. Uno de cada cinco pacientes con algún tipo de "advance directives" no cambiaba de opinión y estaba documentado en el historial médico (16). No debemos de olvidar como destaca von Guten que las decisiones relacionadas con "advances directives" (directrices adelantadas) junto con la comorbilidad, nivel cognitivo estado emocional paciente/familia nos ayudan a determinar la SCET en pacientes con enfermedades avanzadas $(30,39)$.

- Adecuar estrategias de cuidados desde el punto de vista físico, psicológico, espiritual y práctico a cada paciente con 
sus peculiaridades (22). Los modelos de cuidados a los pacientes al final de la vida deben dirigirse hacía los problemas médicos de los pacientes y de sus preferencias. Así los cuidados pueden proporcionar: prevención, prolongación de la vida, rehabilitación y cuidados paliativos.

En el caso de un paciente en que se sospecha que la muerte acontecerá en el transcurso de semanas, meses o incluso años, medidas preventivas como por ejemplo vacunación antigripal puede ayudar a prolongar significativamente la vida. En pacientes encamados durante períodos largos de tiempo, medidas preventivas del encamado pueden prevenir la aparición de úlceras por presión.

Medidas de rehabilitación pueden ser apropiadas en pacientes con capacidad de recuperar niveles de funcionalidad que conllevan a una mejoría de la calidad de vida (CV) y a reducir la carga del cuidador. Aunque no consigan una alta supervivencia, pueden permitir sobrevivir de forma significativa algún período de tiempo (semanas, meses) que puede servir para poder estar presente en acontecimientos familiares importantes previamente planificados.

El manejo adecuado del paciente tiene que tener en consideración los objetivos de los cuidados. Si el objetivo es la prolongación de la vida, el tratamiento de los síntomas tiene que subordinarse a la supervivencia y las intervenciones gravosas pueden ser adecuadas, porque se ajustan a los deseos del paciente. Si el objetivo es una muerte adecuada, el manejo de los síntomas es perseguido vigorosamente aunque comprometa la supervivencia. Intervenciones gravosas tanto diagnosticas como terapéuticas raramente estarían indicadas (Tabla I).

Esta tabla muestra como los cuidados se relacionan con las metas del paciente y familia, estadio de la enfermedad, preferencias, intervenciones, etc. Es razonable pensar que las personas, incluso muy enfermas puede querer conseguir múltiples objetivos: mantener una adecuada imagen, continuar con su rol social, evitar sufrimiento físico, prolongar la vida, etc.

Desde un punto de vista práctico, debemos de tener en cuenta una serie de apartados que nos pueden ayudar a definir la SCET en pacientes con enfermedades avanzadas no

\section{TABLA I}

\section{ÉNFASIS DE LOS CUIDADOS PRIMARIOS CON DIFERENTES} METAS EN ENFERMEDADES QUE AMENAZAN LA VIDA

\begin{tabular}{lccc}
\hline $\begin{array}{l}\text { Aspecto de los } \\
\text { Cuidados }\end{array}$ & Curativo & $\begin{array}{c}\text { Cuidados paliativos } \\
\text { prolongados }\end{array}$ & $\begin{array}{c}\text { Cuidados Paliativos } \\
\text { sintomáticos }\end{array}$ \\
\hline $\begin{array}{l}\text { Impacto sobre la } \\
\text { enfermedad }\end{array}$ & Erradicarla & Frenar progresión & Evitar complicaciones \\
Actitud psicológica & "Canar" & "Luchar" & "Aceptar" \\
Preferencia por RCP & $\mathrm{Si}$ & Probable & No probable \\
Candidato a Hospice & $\mathrm{No}$ & $\mathrm{No}$ & Probable \\
$\begin{array}{l}\text { Prevention/control } \\
\text { síntomas }\end{array}$ & Secundario & Sopesar & Primario \\
Soporte a la familia & $\mathrm{Si}$ & $\mathrm{Si}$ & $\mathrm{Si}$ \\
Planificación anticipada & $\mathrm{Si}$ & $\mathrm{Si}$ & $\mathrm{Si}$ \\
Apoyo al duelo & $\mathrm{No}$ usual & $\mathrm{A} \mathrm{veces}$ & Usualmente \\
\hline
\end{tabular}

Fuente: Adapted from Baines, Gendron, et al., 1996 (22). malignas $(15,24,40,41)$ : a) los factores pronósticos de mortalidad de la enfermedad de base; b) la comorbilidad; c) la situación funcional global; d) la progresión de la enfermedad (declive funcional, hospitalizaciones frecuentes, complicaciones médicas, etc.); e) el estado cognitivo; f) el deterioro nutricional; y g) la presencia de testamento vital o cualquier otra manifestación sobre los cuidados que desea el paciente para el final de su vida.

Además debemos documentar y registrar todos estos parámetros así como las decisiones adquiridas y ofrecer al paciente y familia el tratamiento más adecuado (42). Del mismo modo se necesitan estudios prospectivos que ayuden a definir los criterios de inclusión en programas de CP de paciente con enfermedad crónica avanzada no maligna (43). Con ello podremos proporcionar unos cuidados al final de la vida de calidad y nos acercaremos cada vez más a la definición establecida por The Institute of Medicine Committee on End-of-Life Care sobre la muerte digna: "Muerte libre de distress evitable y sufrimiento para el paciente, familia, cuidadores y en acuerdo con los deseos del paciente y familia y teniendo en cuenta unos estándares clínicos, culturales y éticos" (44). A modo de conclusiones:

Debemos mejorar el conocimiento de los factores pronóstico en determinadas enfermedades avanzadas no malignas así como extender el conocimiento de la medicina paliativa a las diferentes especialidades médicas que atienden a estos enfermos con el fin de que en las últimas etapas de la vida se le proporcione unos CP adecuados.

Una mayor precocidad en la planificación de la toma de decisiones de los pacientes con enfermedades avanzadas, mejorará la implicación de la unidad enfermo-familia y redundará en un mejor control de síntomas evitando situaciones conflictivas en momentos trascendentes de la vida del enfermo.

La percepción de la muerte como fracaso médico y el aferrarse a mantener ciertas actitudes terapéuticas como mecanismo de escape para no afrontar lo inevitable, son dos actitudes que precisan ser modificadas. Para ello, es fundamental aumentar, y en muchísimos casos iniciar, la formación a los profesionales sanitarios de pregrado y de postgrado, fomentando además la investigación, que será la que irá contestando las preguntas, resolviendo las dudas y afianzando las decisiones que surgen a diario en la atención a estos enfermos.

Destacar la importancia de la Valoración Global Integral para reconocer a los pacientes mayores con riesgo de discapacidad y muerte. Dicha valoración nos ayudará a ver globalmente la situación individual del enfermo y a personalizar las decisiones, que deben estar basadas en el juicio clínico apoyado sobre los factores pronósticos citados, así como en las opiniones y deseos del paciente y familia.

Podemos decir que existe un claro reconocimiento de que la Medicina Paliativa es universal en su aplicación, es decir debe abarcar a todos los grupos de edad. La OMS ha establecido que los Cuidados Paliativos constituyen un modelo asistencial adecuado para la mayoría de las situaciones de enfermedad avanzada y Terminal. Estos CP deben abarcar no solo el control de síntomas sino también el apoyo emocional, espiritual, los cuidados de la familia y la preparación para el duelo. 


\section{Bibliografía}

1. Wilson IM, Bunting JS, Curnow R, Knock J. The need for introducing palliative care facilities for non cancer patients. Palliat Med 1994; 8: 181-2.

2. Addington-Hall J, Fakhoury W, McCarthy M. Specialist palliative care in nonmalignant disease. Palliative Med 1998; 12: 417-27.

3. Solano JP, Gomes B, Higginson IJ. A comparison of Symptom Prevalence in far advanced cancer, AIDS, heart disease, chronic pulmonary disease and renal disease. J Pain Symptom Manage 2006; 31: 58-69.

4. Kurti LG, O'Dowd TC. Dying of non-malignant diseases in General Practice. J Palliative Care 1995; 11: 25-31.

5. Navarro Sanz R, Botella Trelis JJ. Cuidados paliativos en enfermedades avanzadas de un órgano. En: Marcos Gómez Sancho (ed). Medicina Paliativa en la Cultura Latina. $1^{a}$ ed. Arán Ediciones S.A., Madrid 1999: 221-31.

6. Farquhar M, Grande G, Todd C, Barclay S. Defining patients as palliative: hospital doctor's versus general practitioners'perceptions. Palliat Med 2002; 16: 247-50.

7. A. Rashid Gatrad, Erica Brown, Aziz Sheikh. Palliative care needs of minorities. Understanding their needs is the key (editorial). BMJ 2003; 327: 176-7.

8. Declaración sobre la atención médica al final de la vida (documento). Organización Médica Colegial y Sociedad Española de Cuidados Paliativos; 2002.

9. WHO Expert Committee. Cancer pain relief and palliative care: Report of a WHO expert committee. Technical report series 804. Geneva: World Health Organization; 1990.

10. Higginson IJ. Evidence based palliative care (editorial). BMJ 1999; 319: 462-63.

11. Lefebreve-Chapiro S, Sebag-Lanoë R. Soins palliatifs chez les personnes âgées. La Revue du Practicien 1999; 49: 1077-80.

12. Gavrin J, Chapman CK. Clinical managment of dying patient in caring for aptients at the end of life (special issue). Wes J Med 1995; 163: 26877.

13. Ley DCH. Select Papers. The First International Conference on the Palliative Care of the Elderly. The Elderly and Palliative Care. J Palliative Care 1989; 5 : 43-58.

14. Cassel CK, Vladeck BC. ICD-9 Code for Palliative or Terminal Care. N Engl J Med 1996; 335: 1232-33.

15. Higginson IJ. Evidence based palliative care (editorial). BMJ 1999; 319: 462-63.

16. The Support Principal Investigator. A controlled trial to improve care for seriously ill hospitalized patients. JAMA 1995; 274: 1591-98.

17. Lynn J, Wilkinson A, Cohn F, Jones SB. Capitated risk-bearing managed care systems could improve end-of-life care. JAGS 1998; 46: 322-30.

18. Kurti LG, O'Dowd TC. Dying of non-malignant diseases in General Practice. J Palliative Care 1995; 11: 25-31.

19. Formiga F, Vivanco V, Cuapio Y, Porta J, Gómez-Batiste X, Pujol R. Morir en el hospital por enfermedad terminal no oncológica: análisis de la toma de decisiones. Med Clin (Barc) 2003; 121: 95-7.

20. Benítez-Rosario MA. Cuidados paliativos en pacientes no oncológicos. Med Clin (Barc) 2003; 121: 297-8.

21. Lynn J. Serving patients who may die soon and their families. The role of Hospice and other services. JAMA 2001; 285: 925-32.

22. Field MJ, Cassel CK. Approaching death. Improving care at the end of life. National Academy Press, Washington, DC; 1997.

23. Billings A. Palliative care. Recent advance. BMJ 2000; 321: 555-8.

24. Incalzi AR, et al. A simple method of recognizing Geriatric patient at risk of death and disability. JAGS 1992; 40: 34-8.

25. Narain P, Rubinstein LZ, Wieland D, Rosbrook B, Strome LS, Pietrusszka F, Morley JE. Predictors of immediate and six month outcomes in hospitalised elderly patients. JAGS 1988; 336: 775-83.

26. San José A, Armadans-Gil L1, Selva A, Jacas C, Solans R, Campos J, Ardanaz J, Vilardell M. Factores predictores de mortalidad y alta a domicilio, en el momento del alta hospitalaria de pacientes ingresados en un Centro Sociosanitario. Rev Gerontol 1997; 7: 217-23.

27. Miralles R. Factores pronósticos en geriatría. Rev Gerontol 1997; 7 : 193-6.

28. Portella E, San José A. Instrumentos de valoración funcional en gerontología. Rev Gerontol 1996; 6: 209-14.

29. Dickinson EJ, Young A. Framework for medical assessment of functional performance. Lancet 1990; 335: 7.

30. von Guten CF, Twaddle Ml. Terminal care for noncnacer patients. Clin Geriatric Med 1996; 12: 349-58.

31. Stuart B, Alexander C, Arenella C, Connnor S, et al. Medical Guidlines for determining prognosis in selected non-cancer diseases. National Hospice Organization. Second Edition. Arlington. 1996.

32. Abrahm JL, Hansen-Flaschen J. Hospice care for patients with advanced lung disease. Chest 2002; 121: 220-9.

33. Shee C.D. Palliation in chronic respiratory disease. Palliat Med 1995; 9 : 3-12.

34. Millane T, Jackson G, Gibbs CR, Lip GYH. ABC of heart failure acute and chronic management strategies. BMJ 2000; 320: 559-62.

35. Gottlieb C F y Butler J. End-of-life care for elderly patients with heart failure. Clinics in Geriatric Medicine 2000; 16: 663-75.

36. Roth K, Lynn J, Zhong Z, Borum M, Dawson NV. Dying with end stage liver disease cirrhosis: Insights from SUPPORT. Study to Understand Prognosis and Preferences for Outcomes and Riks of Treatments. JAGS 2000; 48 (Supl. 5): S122-30.

37. Valderrabano F., Jofre R, López-Gómez JM. Quality of life in end-stage renal disease patients. Am J Kidney Dis 2001; 38: 443-64.

38. Arriola E, González R, Ibarzabal X, Buiza C. Criterios para la inclusión de un paciente con síndrome demencial en la fase asistencial de cuidados paliativos. Rev Esp Geriatr Gerontol 2002; 37: 225-30.

39. Consejería de Salud CCAA de Andalucía. Atención al paciente pluripatológico. ISBN 84:8486-034-5. 2002.

40. Rhymes JA. Clinical management of the terminally ill. Geriatrics, 1991: 46: 57-67.

41. Lefebreve-Chapiro S. Les soins aux personnes âgées. E J Palliative Care 1998; 5: 162-4.

42. JJ Fis et al. End-of-life Decision-Making in the Hospital: Current Prectice and Future Prospects. J Pain Symptom Manage, 1999; 17: 6-15.

43. Bernabeu-Whittel M, García-Movillo AS, González-Becerra C, Ollero M, Fernández A, Cuello-Contreras JA. Impacto de los cuidados paliativos y perfil clínico del paciente con enfermedad Terminal en un área de Medicina Interna. Rev Clin Esp 2006; 206: 178-81.

44. Patrick DL, Randall Curtis J, Engelberg RA, Nielsen E, McCown E. Measuring and improving the quality of Dying and Death. Ann Intern Med 2003; 139: 410-15. 